modes in Toronto, Canada during school drop-off time. Parent surveys were distributed to grades 4-6 classrooms in 20 schools. Parents rated traffic danger en route to school and at school during drop-off as 'not dangerous', or 'dangerous'. Multivariate cluster analyses were conducted.

Results 729 parent surveys were returned with approximately $1 / 4$ of parents reporting either their child's route to school and/or school drop off time were dangerous. Higher measured car speed was a significant determinant of both dangerous route and drop-off. Parent-reported measure of distance and numbers of roads crossed were significantly related to dangerous route. Both ratings of danger were inversely related to reported walking to/ from school.

Significance Car speed was the only objectively measured built environment feature of the school site related to parent's perceptions of traffic danger. The relationship between features of the larger neighbourhood environment surrounding schools and parent perception of traffic danger requires investigation in order to further determine what influences children walking to school.

\section{DETERMINANTS OF PARENT PERCEPTIONS OF DANGEROUS TRAFFIC RELATED TO SCHOOL TRAVEL}

doi:10.1136/injuryprev-2012-040580g.32

${ }^{1} \mathrm{~L}$ Rothman*, ${ }^{1} \mathrm{C}$ Macarthur, ${ }^{1} \mathrm{~T}$ To, ${ }^{2} \mathrm{R}$ Buliung, ${ }^{1} \mathrm{~A}$ Howard. ${ }^{1}$ The Hospital for Sick Children, Canada; ${ }^{2}$ University of Toronto, Canada

Background Walking to/from school is an important source of physical activity for children. Parents' perceptions of dangerous traffic affect whether or not their child walks to/from school. The determinants of parent's perceptions of dangerous traffic are currently unknown.

Aims/Objectives/Purpose To determine which objective measures of the built environment and traffic at elementary schools are related to parent's perceptions of traffic danger.

Methods Trained observers conducted school site surveys, vehicle speed/volume measurements and counts of school transportation 